\title{
Superparamagnetic reconstituted high-density lipoprotein nanocarriers for magnetically guided drug delivery
}

This article was published in the following Dove Press journal:

International Journal of Nanomedicine

22 February 2017

Number of times this article has been viewed

\author{
Sarika Sabnis' \\ Nirupama A Sabnis' \\ Sangram Raut ${ }^{2}$ \\ Andras G Lackol,3 \\ 'Institute of Cardiovascular and \\ Metabolic Diseases, University of \\ North Texas Health Science Center, \\ ${ }^{2}$ Department of Physics, Texas \\ Christian University, ${ }^{3}$ Department of \\ Pediatrics, University of North Texas \\ Health Science Center, Fort Worth, \\ TX, USA
}

\begin{abstract}
Current cancer chemotherapy is frequently associated with short- and long-term side effects, affecting the quality of life of cancer survivors. Because malignant cells are known to overexpress specific surface antigens, including receptors, targeted drug delivery is often utilized to reduce or overcome side effects. The current study involves a novel targeting approach using specifically designed nanoparticles, including encapsulation of the anti-cancer drug valrubicin into superparamagnetic iron oxide nanoparticle (SPION) containing reconstituted high-density lipoprotein (rHDL) nanoparticles. Specifically, rHDL-SPION-valrubicin hybrid nanoparticles were assembled and characterized with respect to their physical and chemical properties, drug entrapment efficiency and receptor-mediated release of the drug valrubicin from the nanoparticles to prostate cancer (PC-3) cells. Prussian blue staining was used to assess nanoparticle movement in a magnetic field. Measurements of cytotoxicity toward PC-3 cells showed that rHDLSPION-valrubicin nanoparticles were up to 4.6 and 31 times more effective at the respective valrubicin concentrations of $42.4 \mu \mathrm{g} / \mathrm{mL}$ and $85 \mu \mathrm{g} / \mathrm{mL}$ than the drug valrubicin alone. These studies showed, for the first time, that lipoprotein drug delivery enhanced via magnetic targeting could be an effective chemotherapeutic strategy for prostate cancer.
\end{abstract}

Keywords: drug delivery, nanoparticles, rHDL, magnetic nanoparticles, SPION

\section{Background}

Despite many new approaches involving gene and immunotherapy, surgery and subsequent adjuvant chemotherapy remain the standard clinical approaches for the management of most malignancies, the second highest cause of mortality worldwide. ${ }^{1-5}$ The efficacy of cancer chemotherapy is often limited by physical and biological barriers. ${ }^{6-9}$ These include solubility, bioavailability, pharmacokinetics and biological barriers such as tumor microenvironment, hypoxic core and extracellular tumor $\mathrm{pH}$, interstitial fluid pressure and many other factors. ${ }^{9-15}$ Nanotechnology has been invoked as a means of overcoming several of these difficulties. ${ }^{16}$ More specifically, cancer chemotherapy is frequently associated with short- and long-term side effects, ${ }^{17-20}$ affecting the quality of life of cancer survivors. ${ }^{21,22}$ To advance the state of the art in cancer therapeutics, numerous new approaches have been developed, including active or passive targeting of the drug to the desired site via nanocarrier drug delivery platform designed as transporters of anti-cancer drugs with the potential application for enhanced chemotherapy. ${ }^{23-26}$ Other localized treatment options include magnetically guided drug delivery utilizing iron particles that can subsequently be directed to the site of the tumor..$^{27,28}$

The current study involves a novel targeting approach utilizing encapsulation of the anti-cancer drug into reconstituted high-density lipoprotein (rHDL) nanoparticles with
Correspondence: Andras G Lacko Institute of Cardiovascular and metabolic Diseases, University of North Texas Health Science Center, 3500 Camp Bowie Blvd, Fort Worth, TX 76I07, USA Email andras.lacko@unthsc.edu (c)
hereby accept the Terms. Non-commercial uses of the work are permitted without any further permission from Dove Medical Press Limited, provided the work is properly attributed. For permission for commercial use of this work, please see paragraphs 4.2 and 5 of our Terms (https://www.dovepress.com/terms.php). 
superparamagnetic iron oxide nanoparticle (SPION). rHDLs and other lipoprotein constructs have been developed by combining apolipoprotein A-1 (Apo-A1) or its mimetic peptides with phospholipids. ${ }^{29-33}$ The resultant lipoprotein mimetic nanoparticles possess several favorable characteristics for drug transport, including their small size, biocompatibility, stability, monodispersity and high drug payload. In addition, the lipid and protein components of rHDL may be modified via conjugation with targeting molecules to optimize their pharmaceutical potential for clinical applications. ${ }^{33,34}$ Earlier studies from our laboratory demonstrated that the solubility and bioavailability of valrubicin may be enhanced by incorporation into rHDL nanoparticles..$^{29,30,33,34}$ In addition, Shah et $\mathrm{al}^{35}$ provided proof of concept via photophysical characterization of the rHDL-valrubicin complex as a potential tumor imaging agent.

These preclinical studies were designed to evaluate the effectiveness of SPIONs in combination with the drug valrubicin (AD-32) carrying rHDL nanoparticles as potential cancer therapeutic agents. Some of the physical/chemical properties of the SPION-rHDL/valrubicin hybrid nanoparticles have been evaluated, in addition to their cytotoxicity against prostate cancer cells and their interaction with the cancer cells via a scavenger receptor-mediated (scavenger receptor type B [SR-B1]) mechanism. These studies showed, for the first time, that lipoprotein drug delivery enhanced via magnetic targeting could be an effective chemotherapeutic strategy for prostate cancer.

\section{Materials and methods Materials}

All reagents were purchased from Sigma-Aldrich (St Louis, MO, USA). Prussian blue was purchased from Acros Organics (Geel, Belgium). All organic chemicals and solvents used were of reagent grade. Bacterial protein extraction reagent and bicinchoninic acid (BCA) protein assay kits were purchased from Thermo Fisher Scientific (Rockford, IL, USA). Apo-A1 was obtained from Mc Lab (San Francisco, CA, USA). Cholesterol and phospholipid estimation kits were obtained from Wako Pure Chemical Industries Ltd (Richmond, VA, USA). Block lipid transport-1 (BLT-1; SR-B1 inhibitor) was obtained from Cambridge Corporation (San Diego, CA, USA) and prepared as $5 \mathrm{mg} / \mathrm{mL}$ stock solution in $100 \%$ dimethyl sulfoxide (DMSO). Roswell Park Memorial Institute (RPMI) 1640 media, keratinocyte media and fetal bovine serum (FBS) were obtained from Invitrogen (Carlsbad, CA, USA). A prostate cancer cell line PC-3 and an immortalized normal epithelial cell line PZ-HPV were obtained from American Type Culture Collection (ATCC; Manassas, VA, USA).

\section{Methods}

\section{Preparation of SPIONs}

SPION nanoparticles were prepared as described by Raut et al. ${ }^{36}$ Thermal decomposition was used to prepare oleic acid-coated magnetic nanoparticles (OAMNP). A total of $10 \mathrm{~mL}$ of octyl ether was added to $1.43 \mathrm{~mL}$ of oleic acid. The mixture was heated to $100^{\circ} \mathrm{C}$ under an inert atmosphere for 30 minutes. To this mixture, $0.28 \mathrm{~mL}$ of iron pentacarbonyl $\left[\mathrm{Fe}(\mathrm{CO})_{5}\right]$ was added, followed by a raise in the temperature to $300^{\circ} \mathrm{C}$, which was maintained until the reaction mixture turned black. The solution was then allowed to cool down to room temperature, after which ethanol was added to precipitate the nanoparticles. The mixture was then centrifuged at 8,000 rpm for 15 minutes, after which the obtained precipitate was re-dissolved in hexane. This process of washing the OAMNP with hexane was repeated three times, and the nanoparticles were stored as hexane dispersion until further use.

\section{Preparation of rHDL nanoparticles}

The rHDL nanoparticles containing drug and SPIONs were prepared via the cholate dialysis procedure with some modifications. ${ }^{33,34}$ To a glass vial, phosphatidylcholine $7.5 \mathrm{mg} / \mathrm{mL}$, free cholesterol $0.175 \mathrm{mg} / \mathrm{mL}$ and cholesteryl oleate $0.075 \mathrm{mg} / \mathrm{mL}$, all from previously prepared stocks, were added and mixed thoroughly. This mixture was then dried under nitrogen. To this mixture, $1 \mathrm{mg} / \mathrm{mL}$ anti-cancer drug (valrubicin) and $30 \mu \mathrm{L} / \mathrm{mL}$ DMSO were added, followed by $2.5 \mathrm{mg} / \mathrm{mL}$ Apo-A1 and $1 \mathrm{mg} / \mathrm{mL}$ SPIONs (wet weight). The mixture was then vortexed. After adding $7.5 \mathrm{mg} / \mathrm{mL}$ sodium cholate, buffer $(10 \mathrm{mM}$ Tris, $0.1 \mathrm{M}$ potassium chloride, $1 \mathrm{mM}$ ethylenediaminetetraacetic acid [EDTA] $\mathrm{pH}$ 8.0) was used to make up the desired volume. The mixture was mixed thoroughly, frozen and then lyophilized overnight. It was then dialyzed against $2 \mathrm{~L}$ of $1 \times$ phosphate-buffered saline (PBS) for 48 hours, with a change of buffer three times every 2 hours on the 1st day, and later kept overnight. The mixture was centrifuged at 1,500 rpm for 1 minute and then filtered through a $0.45-\mu \mathrm{M}$ filter and stored at $4^{\circ} \mathrm{C}$ until used (Figure 1).

\section{Estimation of chemical composition of nanoparticles}

Chemical composition estimations were determined using respective chemical assays, which were conducted using 96-well microtiter plates. Measurements of absorbance were conducted at different wavelengths (as outlined in each assay procedure) using a PowerWave 340 spectrophotometer 


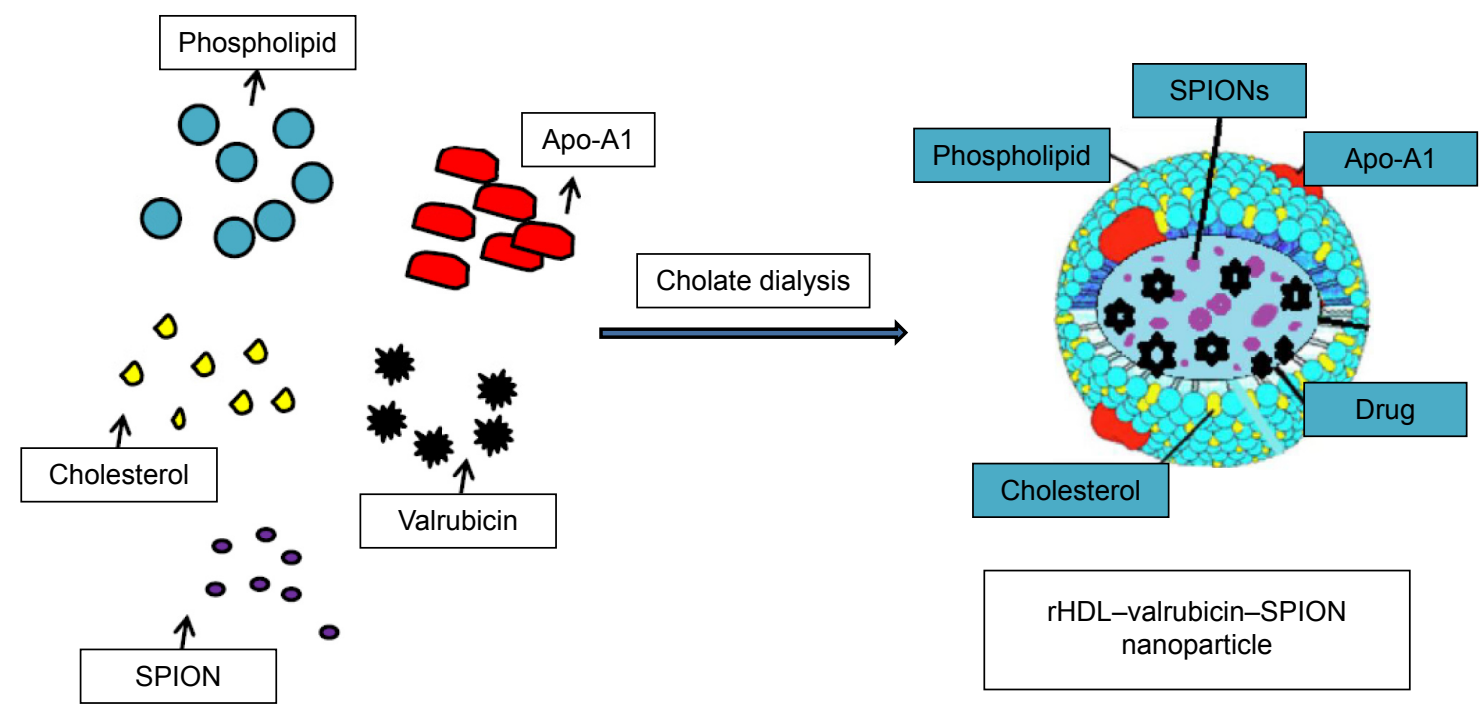

Figure I Schematic representation of formulation of rHDL-valrubicin-SPION nanoparticles - a conceptual model.

Abbreviations: $\mathrm{rHDL}$, reconstituted high-density lipoprotein; SPION, superparamagnetic iron oxide nanoparticle; Apo-AI, apolipoprotein A-I.

(Bio-Tek, Winooski, VT, USA). Standard graphs were then plotted with the known concentrations of respective standards, and the concentrations of each component were calculated. Each assay was carried out at least twice, and estimations for samples were carried out in triplicates. The following chemical composition estimations were conducted:

1. Cholesterol and phospholipid contents were determined by their respective enzymatic reagent kits (Cholesterol E and Phospholipid C; Waco Chemicals, Richmond, VA, USA) as per manufacturer's suggestions.

2. Protein estimations were conducted using a BCA protein assay kit as per manufacturer's instructions. A total of $10 \mu \mathrm{L}$ of each sample was placed in triplicate, and $200 \mu \mathrm{L}$ of BCA reagent was added and mixed thoroughly. The plates were incubated at $37^{\circ} \mathrm{C}$ for 30 minutes and then read at $540 \mathrm{~nm}$.

3. Drug concentration was monitored by measuring the absorbance at $490 \mathrm{~nm}$. The concentrations were extrapolated from a standard curve using different concentrations of valrubicin. Three individual readings were taken, and average and standard deviation (SD) were calculated.

4. Iron incorporation into the rHDL nanoparticles was assessed by using the phenanthroline reagent with some modifications adapting the assay to be carried out in a 96-well plate. ${ }^{37}$ Ferrous ammonium sulfate hexahydrate solution $(7 \mathrm{mg} / \mathrm{mL})$ was used to prepare the standard curve for the iron determinations. Briefly, aliquots of the standard stock solution ranging from $1 \mu \mathrm{L}$ to $20 \mu \mathrm{L}$ were placed. To this solution, $1 \mu \mathrm{L}$ of hydroxyl amine from stock solution $(100 \mathrm{mg} / \mathrm{mL}), 10 \mu \mathrm{L}$ of 1,10 -phenanthroline solution (from a stock of $1 \mathrm{mg} / \mathrm{mL}$ ) and $8 \mu \mathrm{L}$ of sodium acetate (from a stock of $1.2 \mathrm{M}$ ) was sequentially added. Finally, the samples were mixed and incubated at room temperature for 10 minutes, before measuring their absorbance at $450 \mathrm{~nm}$.

\section{Entrapment efficiency of iron and valrubicin}

The drug or iron entrapment efficiency (EE) was determined using the equation:

$$
\mathrm{EE}=\frac{\text { Final Drug or Iron Concentration }}{\text { Initial Drug or Iron Concentration }} * 100
$$

\section{Measurement of size and zeta potential of the nanoparticles}

The hydrodynamic diameter of the nanoparticles was estimated by dynamic laser scattering with a Delsa Nanoparticle Size Analyzer (Malvern Instruments, Herrenberg, Germany). An average of 70 runs was captured by the machine and is indicated by the average particle size. Zeta potential was measured using Zetasizer Nano ZS (Malvern Instruments). The zeta potential measurements were performed by dispersing the particles in an aqueous solution at $25^{\circ} \mathrm{C}$ with a scattering angle of $90^{\circ}$. The experiment was repeated three times, and the results were averaged.

\section{Demonstration of morphology of rHDL-SPION particles using transmission electron microscopy (TEM)}

Nanoparticles were imaged using TEM by depositing nanoparticles on a TEM grid from a PBS dispersion. After drying, 
$1 \%$ phosphotungstic acid as a negative stain was applied and TEM grids were left to dry at room temperature. The particles were then imaged using a JEM 2100 transmission electron microscope (JEOL, Peabody, MA, USA).

\section{Uptake of iron and valrubicin in presence of SR-BI inhibitors}

Prostate cancer cells (PC-3) were plated in two 24-well plates $(100,000$ cells/well) in the RPMI medium with $10 \%$ FBS and incubated at $37^{\circ} \mathrm{C}$ with $5 \% \mathrm{CO}_{2}$ for 24 hours. The monolayers were washed with PBS, pH 7.4, and then incubated at $37^{\circ} \mathrm{C}$ with serum-free medium for 90 minutes. The cells were washed with PBS, and serum-free medium was again added. To one of the plates, $5 \mu \mathrm{M}$ of BLT-1 was added in each well, and to another plate, $10 \mu \mathrm{g} / \mathrm{mL}$ of human high-density lipoprotein (HDL) was added. The plates were incubated for 30 minutes at $37^{\circ} \mathrm{C}$. Two wells were kept as no inhibitor control. To one of the plates, $20 \mu \mathrm{L}$ of the rHDL-SPION was added to each well, and to the other plate, $20 \mu \mathrm{L}$ of rHDL-SPION-valrubicin nanoparticles were added. The plates were incubated at $37^{\circ} \mathrm{C}$ for 90 minutes. The wells were washed once with $1 \times \mathrm{PBS}, \mathrm{pH} 3.0$, and subsequently with $1 \times \mathrm{PBS}, \mathrm{pH} 7.4$, respectively. The cells were then lyzed with lysis buffer (50 mM Tris-HCl [pH 8.0], $150 \mathrm{mM} \mathrm{NaCl,} \mathrm{0.02 \%}$ sodium azide, $100 \mathrm{mg} / \mathrm{mL}$ phenylmethylsulfonyl fluoride, $1 \mathrm{mg} / \mathrm{mL}$ aprotinin and $1 \%$ Triton X-100). The lysates were centrifuged at 10,000 rpm for 5 minutes. The protein content of the lysate was determined by BCA assays. The iron content and valrubicin content were measured by an iron estimation assay and spectrophotometric measurements, respectively.

\section{Monitoring the movement of the rHDL-SPION particles}

PC-3 cells were grown on a Petri dish. The cells were treated with $20 \mu \mathrm{L}$ of rHDL-SPION particles. A magnetic force was applied at one end of the Petri dish to attract SPIONs. The plates were incubated at $37^{\circ} \mathrm{C}$ for 5 minutes. The cells were then washed with PBS ( $\mathrm{pH} 7.4$ ), keeping the magnet attached at the end. Equal parts of $20 \% \mathrm{HCl}$ and $10 \%$ potassium ferrocyanide (Prussian blue) were prepared immediately before use. ${ }^{38}$ The plate was covered in this solution for 20 minutes, washed three times with distilled water, counterstained with nuclear fast red for 5 minutes and rinsed twice with distilled water. Images were taken at areas near and away from the magnetic field using a TMS microscope (Nikon Instruments Inc., Melville, NY, USA) with camera.

\section{Stability of rHDL-SPION and rHDL-SPION- valrubicin nanoparticles}

Stability studies were conducted by the method described earlier. ${ }^{39}$ A total of $1 \mathrm{~mL}$ of rHDL-SPION and rHDLSPION-valrubicin formulation was placed in a dialysis bag (6-8 kD molecular weight cut off). The bags were placed in $50 \mathrm{~mL}$ of $1 \times \mathrm{PBS}$ and incubated at $37^{\circ} \mathrm{C}$ for 48 hours. A total of $100 \mu \mathrm{L}$ of the buffer was withdrawn at 0-, 2-, 4-, 6-, 8-, 24- and 48-hour intervals. Each time the buffer was withdrawn, a fresh solution of $100 \mu \mathrm{L}$ of PBS was transferred in the outer chamber. A sample was saved before starting the dialysis, and a sample inside the dialysis bag was collected after the incubation period was over. Iron and valrubicin concentrations of the respective samples were determined by appropriate assay methods.

\section{Cytotoxicity studies using 3-(4,5-dimethylthiazol- 2-yl)-2,5-diphenyltetrazolium bromide (MTT) assay method}

A standard protocol using 5,000 cells/well of a 96-well plate was used. ${ }^{40} \mathrm{PC}-3$ and PZ-HPV cells were incubated at $37^{\circ} \mathrm{C}$ in RPMI-1640 and keratinocyte media, respectively, and treated with increasing drug concentrations. The plate was divided into two parts: one part was treated with free valrubicin at concentrations of $0.85 \mu \mathrm{g} / \mathrm{mL}$, $8.5 \mu \mathrm{g} / \mathrm{mL}, 17 \mu \mathrm{g} / \mathrm{mL}, 42.5 \mu \mathrm{g} / \mathrm{mL}$ and $85 \mu \mathrm{g} / \mathrm{mL}$ and the other part was treated with the same concentration of rHDL-SPION-valrubicin. For each concentration, four replicates were used; for control, cells without drug were used as a control. In a separate plate, rHDL and rHDLSPION were each tested with corresponding varying protein concentrations. A control with rHDL-valrubicin was also kept. The plates were incubated for 48 hours at $37^{\circ} \mathrm{C}$. A total of $25 \mu \mathrm{L}$ of MTT reagent was added to each well, and plates were incubated at $37^{\circ} \mathrm{C}$ for 3 hours. The plates were centrifuged at 1,500 rpm for 5 minutes, and the supernatant was then discarded. The purple-colored compound in the resulting pellet was dissolved in DMSO, and the absorbance was measured at $570 \mathrm{~nm}$. Percentage survival of cells was calculated and plotted against concentration of valrubicin.

\section{Statistical analysis}

Data of EE, mean diameter and zeta potential are reported as mean \pm SD. Percentage iron uptake, percentage iron retained and percentage cell survival are reported as mean \pm standard error of the mean. The half maximal inhibitory concentration $\left(\mathrm{IC}_{50}\right)$ values for the different drug formulations were calculated 
using CalcuSyn software (Cambridge, UK). The results were analyzed using analysis of variance (ANOVA), and the differences were considered significant at a level of $P<0.05$.

\section{Results}

\section{Compositional analysis}

Chemical composition of empty nanoparticles (Figure 2A), rHDL-SPION nanoparticles (Figure 2B), rHDL-valrubicin nanoparticles (Figure 2C) and rHDL-SPION-valrubicin nanoparticles (Figure 2D) are shown. The percentage distribution of phospholipid, protein and cholesterol of the rHDL, rHDL-SPION, rHDL-valrubicin and rHDL-SPION-valrubicin complexes ranged from $41.11 \%$ to $53.72 \%, 32.56 \%$ to $46.83 \%$ and $5.54 \%$ to $8.53 \%$, respectively, indicating that the incorporation of SPION does not substantially alter the chemical composition of the rHDL/drug formulation. The amounts of iron in rHDLSPION and rHDL-SPION-valrubicin nanoparticles were $0.75 \%$ and $0.61 \%$, respectively, whereas the valrubicin content was $\sim 3 \%$ lower in the rHDL-SPION-valrubicin nanoparticles compared to the rHDL-valrubicin nanoparticles (Figure 2B-D).

\section{Iron and valrubicin EE}

The entrapment of valrubicin in the rHDL-valrubicin nanoparticles vs rHDL-SPION-valrubicin nanoparticles was $89 \%$ and $55 \%$, respectively (Figure 3A). The EE of iron was $44 \%$ in rHDL-SPION nanoparticles and 30\% in rHDL-SPIONvalrubicin nanoparticles (Figure 3B). As may be seen in the following data (Figure 3A and B).
A

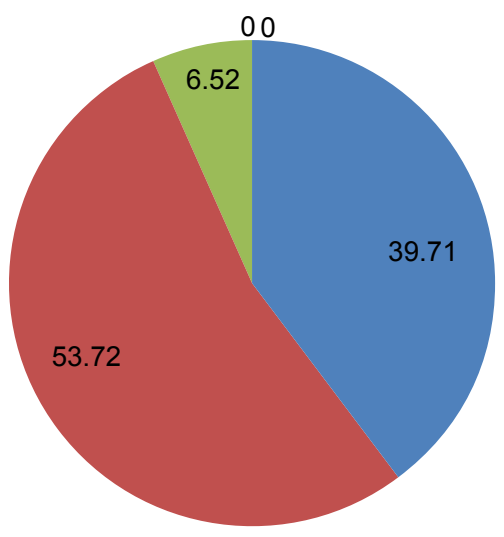

B

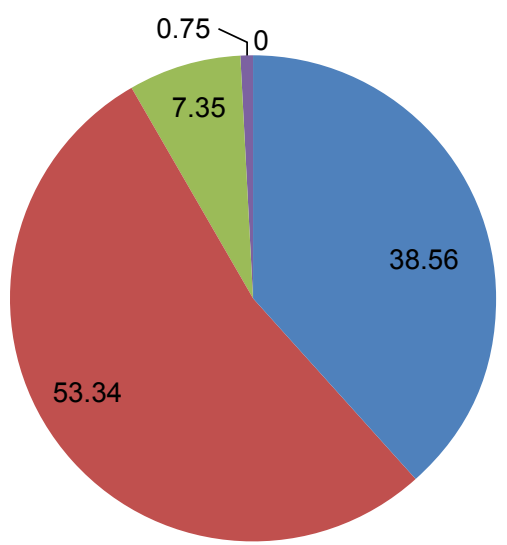

C

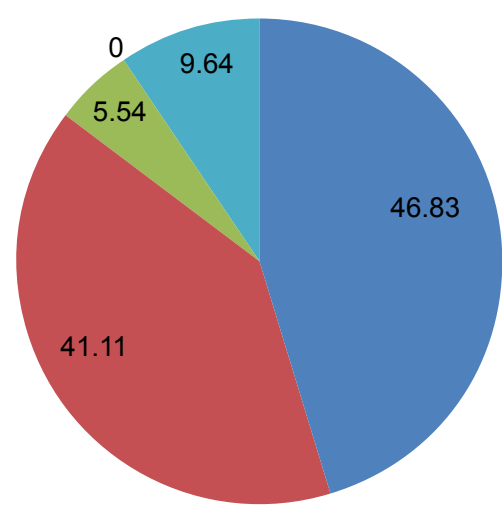

D

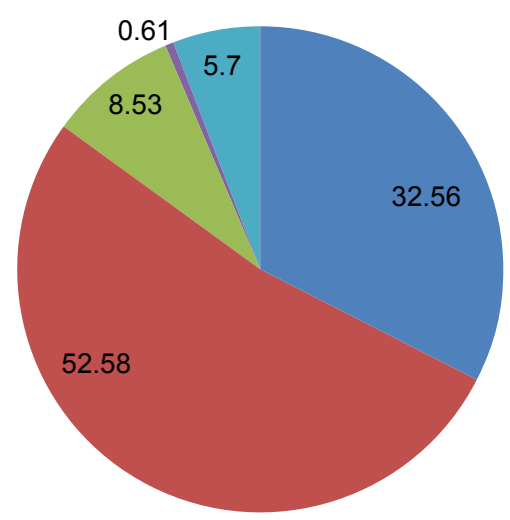

Protein Phospholipid Cholesterol Iron Valrubicin

Figure 2 Chemical compositional analysis of nanoparticles.

Notes: (A) rHDL only; (B) rHDL-SPION; (C) rHDL-Valrubicin; (D) rHDL + SPION + valrubicin. Protein (dark blue), phospholipid (red), cholesterol (green), iron (purple) and valrubicin (light blue) were estimated by standard procedures.

Abbreviations: rHDL, reconstituted high-density lipoprotein; SPION, superparamagnetic iron oxide nanoparticle. 
A

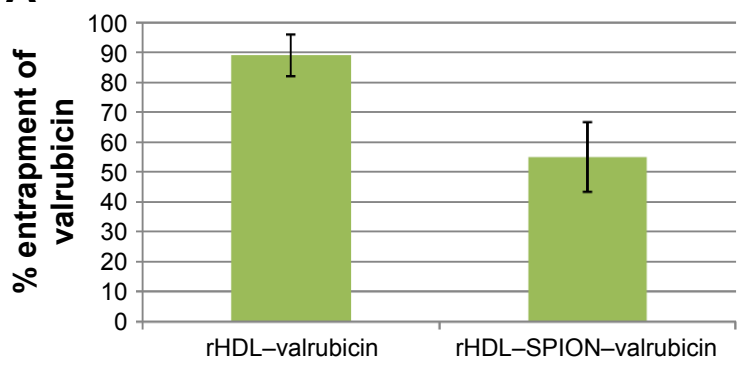

B

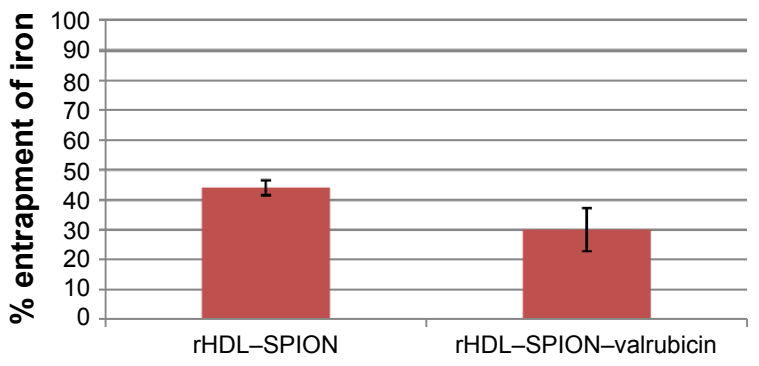

Figure $3 \mathrm{EE}$ in rHDL-valrubicin, rHDL-SPION and rHDL-SPION-valrubicin: (A) valrubicin and (B) iron.

Note: The bars represent the amount of material incorporated relative to the respective initial amounts present.

Abbreviations: EE, entrapment efficiency; rHDL, reconstituted high-density lipoprotein; SPION, superparamagnetic iron oxide nanoparticle.

\section{Physical properties of the nanoparticles}

The particle diameter analysis of the four types of nanoparticles showed them to be reasonably homogeneous, while the average diameter increased with the addition of SPIONs and/or valrubicin (presumably located) in the core of the nanoparticles (Table 1 and Figure 4A-D). The average diameter of rHDL, rHDL-SPION, rHDL-valrubicin and rHDL-SPION-valrubicin was $7.1 \pm 1.3 \mathrm{~nm}, 67.6 \pm 19.4 \mathrm{~nm}$, $48.1 \pm 13.9 \mathrm{~nm}$ and $96.9 \pm 18.7 \mathrm{~nm}$, respectively. The polydispersity index (PDI) of these particles was $0.47,0.352,0.350$ and 0.378 , respectively.

Zeta potential was also assessed as an indication of the electrostatic or charge repulsion/attraction between particles and as a potential barrier to cell/nanoparticle interactions. ${ }^{40,41}$ The zeta potential values for these particles are shown in Table 1. Insertion of the drug (valrubicin) into the core of the respective nanoparticles ${ }^{35}$ changed the zeta potential from $-10.57 \pm 0.157$ for the rHDL nanoparticles to $-7.93 \pm 0.548$ for the rHDL-SPION-valrubicin nanoparticles.

\section{Receptor-mediated uptake of the iron payload from the nanoparticles}

The uptake of SPIONs and the drug from rHDL-SPION and rHDL-SPION-valrubicin nanoparticles was anticipated to proceed via the SR-B1 receptors. ${ }^{32,42}$ To investigate this

Table I Comparative physical characteristics of rHDL nanoparticles

\begin{tabular}{llll}
\hline Type of particles & $\begin{array}{l}\text { Average } \\
\text { diameter } \pm \text { SD }\end{array}$ & PDI & $\begin{array}{l}\text { Zeta } \\
\text { potential } \pm \text { SD }\end{array}$ \\
\hline rHDL only & $7.1 \pm I .3$ & 0.470 & $-10.57 \pm 0.153$ \\
rHDL-SPION & $67.6 \pm 19.4$ & 0.352 & $-8.61 \pm 0.726$ \\
rHDL-valrubicin & $48.1 \pm 13.9$ & 0.350 & $-8.27 \pm 0.724$ \\
rHDL-SPION-valrubicin & $96.9 \pm 18.7$ & 0.378 & $-7.93 \pm 0.548$ \\
\hline
\end{tabular}

Note: Average particle size, PDI and zeta potential were measured.

Abbreviations: rHDL, reconstituted high-densitylipoprotein; SD, standard deviation; PDI, polydispersity index; SPION, superparamagnetic iron oxide nanoparticle. uptake mechanism, two inhibitors of SR-B1 were selected (BLT-1 and human HDL) for the iron uptake studies. Incubations in the absence of the SR-B1 inhibitor were considered as $100 \%$ iron uptake. The average iron uptake from the rHDL-SPION nanoparticles was reduced to $12.2 \%$ and $15.4 \%$ (of the control), respectively, in the presence of $5 \mu \mathrm{M}$ BLT-1 and $10 \mu \mathrm{g} / \mathrm{mL}$ HDL (Figure 5A). The same trend was observed with rHDL-SPION-valrubicin nanoparticles in the presence of BLT-1 and HDL, resulting in decreased iron uptakes to $20.1 \%$ and $18.2 \%$ of the (uninhibited) control, respectively (Figure 5B).

\section{Movement of SPIONs in response to a magnetic field}

This experiment was designed to determine whether the SPIONs containing nanoparticles move in the direction of the magnetic field (Figure 6A). When the SPIONs containing rHDL nanoparticles were incubated on a plate grown with prostate cancer cells (PC-3), the cells in the vicinity of the applied magnetic field (Figure 6B) showed high iron particle concentration, as shown by the deep blue color due to Prussian blue dye staining. The cells on the opposite end of the magnetic field did not incorporate the SPIONs (Figure 6C), as evidenced by the lack of blue color, indicating that only the SPIONs containing nanoparticles may be maneuvered to a desired location via a magnetic field.

\section{Stability of rHDL-SPION and rHDL-SPION-valrubicin}

The stability of rHDL-SPION and rHDL-SPION-valrubicin was assessed under conditions resembling a physiological milieu $\left(\mathrm{pH} 7.4,37^{\circ} \mathrm{C}\right)$. The initial iron content was designated as $100 \%$. After 48 hours, rHDL-SPION had $67.07 \%$ of iron remaining, while rHDL-SPION-valrubicin had $65.5 \%$ of iron remaining (Figure 7). 
A
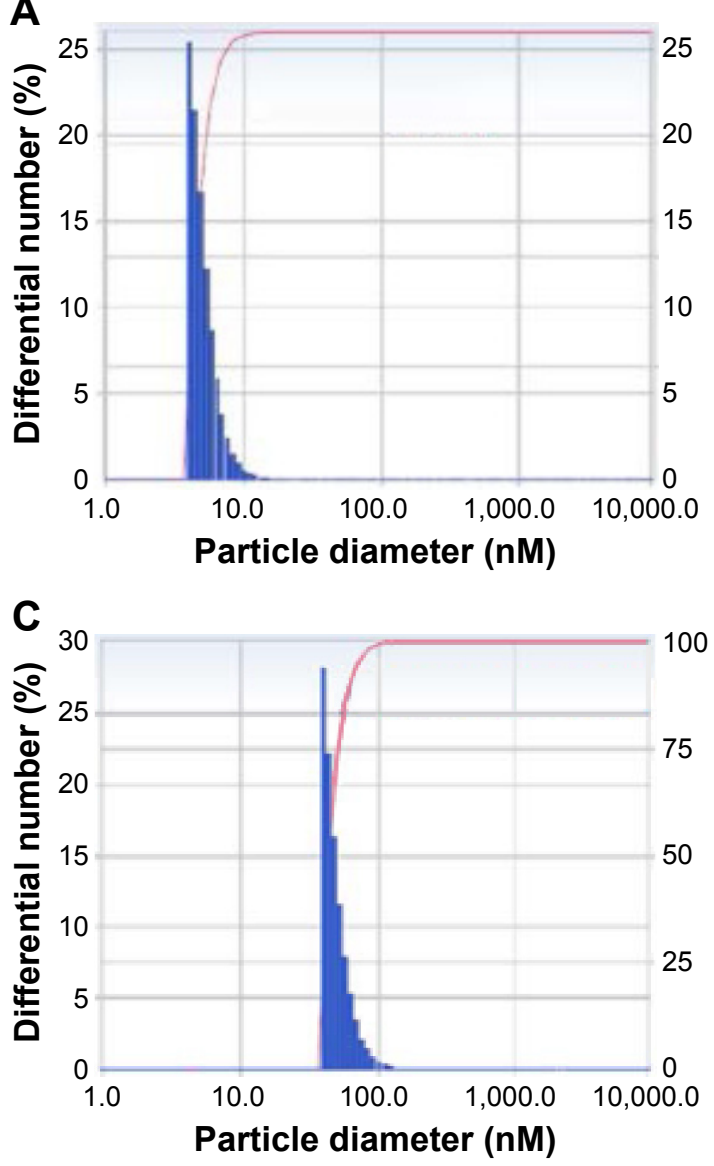

B

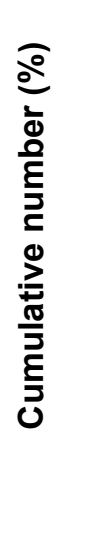

100

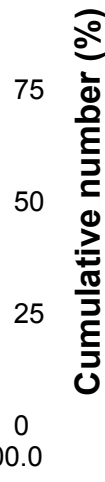

Particle diameter (nM)

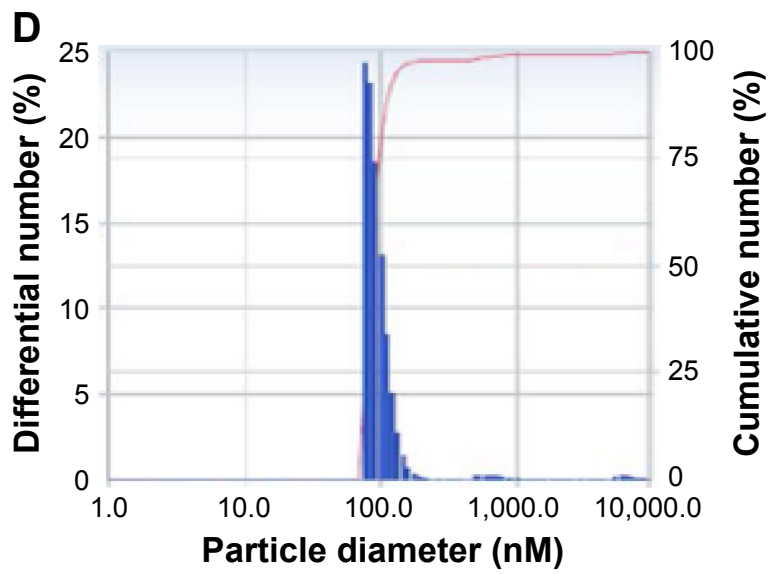

Figure 4 Comparative size of rHDL nanoparticles.

Notes: (A) rHDL only, (B) rHDL-SPION, (C) rHDL-valrubicin and (D) rHDL-SPION-valrubicin. Measurements were taken using Delsa Nanoparticle Size Analyzer. Abbreviations: $\mathrm{rHDL}$, reconstituted high-density lipoprotein; SPION, superparamagnetic iron oxide nanoparticle.

Cytotoxicity of free valrubicin and rHDL-SPION-valrubicin against (malignant) prostate cancer cells (PC-3) and (nonmalignant) prostate epithelial cells (PZ-HPV)

The cytotoxicity of valrubicin delivered via rHDL-SPION nanoparticles was compared to free (unencapsulated) valrubicin. A concentration-dependent decrease in the survival of PC-3 and PZ-HPV cells was observed with both free and rHDL-encapsulated valrubicin preparations (Figure 8A and $\mathrm{B})$. The effect of rHDL-SPION-valrubicin preparation on PC-3 cell survival was most pronounced at higher valrubicin concentrations, producing $92 \%$ and $100 \%$ killing, respectively, at $42.4 \mu \mathrm{g} / \mathrm{mL}(58.5 \mu \mathrm{M})$ and $85 \mu \mathrm{g} / \mathrm{mL}(117.5 \mu \mathrm{M}$;
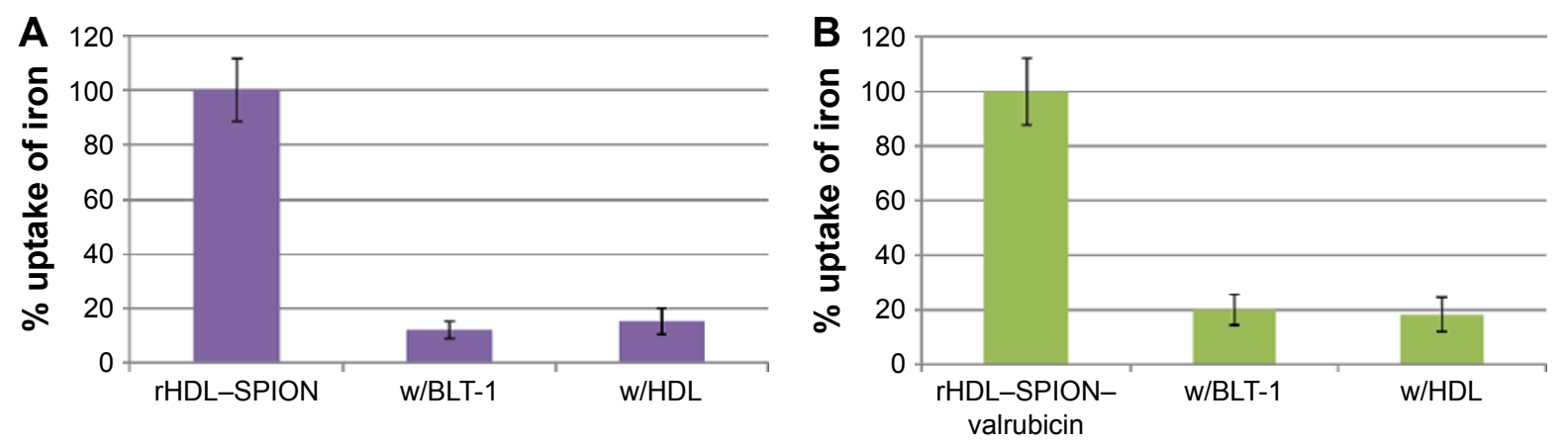

Figure 5 Inhibition of iron uptake from rHDL-SPION (A) and rHDL-SPION-valrubicin (B) nanoparticles by PC-3 cells, induced by a BLT-I inhibitor and an excess of human HDL.

Abbreviations: rHDL, reconstituted high-density lipoprotein; SPION, superparamagnetic iron oxide nanoparticle; BLT-I, block lipid transport-I; HDL, high-density lipoprotein; w/, with. 

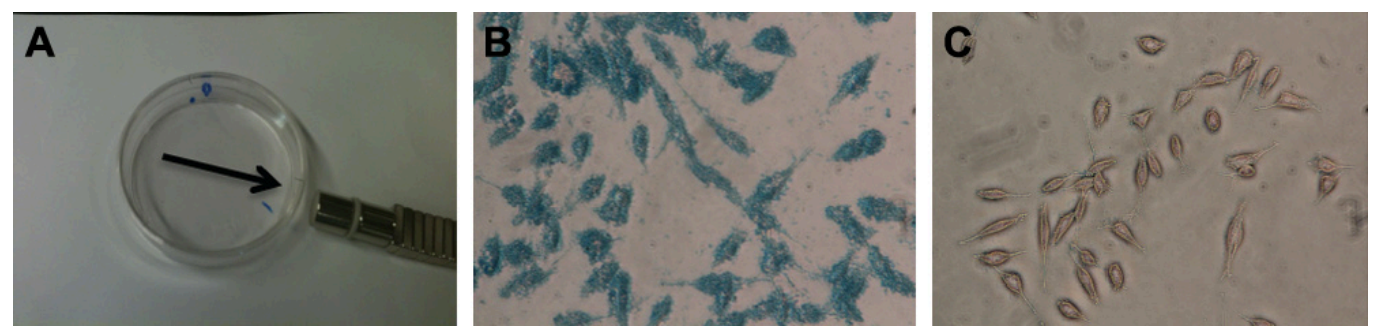

Figure 6 Movement of SPIONs in response to magnetic field.

Notes: Prussian blue staining shows location of iron particles with respect to cancer cells. (A) Petri dish with prostate cancer cells treated with rHDL-SPION nanoparticles to which magnetic field is applied. (B) Cells closer to magnetic field. (C) Cells away from magnetic field (did not turn blue with stain). Magnification $\times 20$.

Abbreviations: SPION, superparamagnetic iron oxide nanoparticle; rHDL, reconstituted high-density lipoprotein.

Figure 8A). On the other hand, the free drug administered at the same concentrations inhibited PC-3 survival by only $60 \%$ and $79 \%$, respectively. (The control rHDL and rHDLSPION nanoparticles with the same amounts of protein showed $<10 \%$ inhibition at these higher concentrations, data not shown). The $\mathrm{IC}_{50}$ values for free valrubicin and rHDLSPION-valrubicin were found to be $16 \mu \mathrm{g} / \mathrm{mL}$ and $12 \mu \mathrm{g} / \mathrm{mL}$ (22.1 $\mu \mathrm{M}$ and $16.5 \mu \mathrm{M})$, respectively $(P$-value $<0.05)$. On the other hand, an opposite trend was observed regarding the survival of nonmalignant PZ-HPV cells in the presence of free valrubicin vs rHDL-SPION-valrubicin. At the respective valrubicin concentrations of $42.4 \mu \mathrm{g} / \mathrm{mL}$ and $85 \mu \mathrm{g} / \mathrm{mL}$, the percentage survival of cells treated with rHDL-SPIONvalrubicin nanoparticles was twice as compared to those

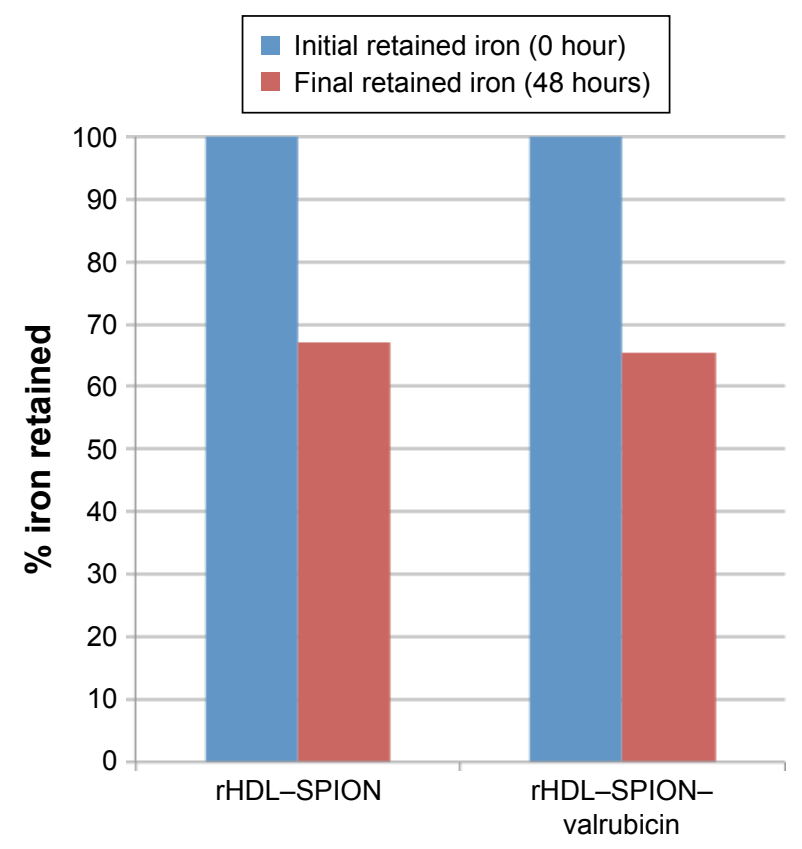

Figure 7 Stability of rHDL-SPION-valrubicin nanoparticles.

Notes: Samples were dialyzed against PBS at $\mathrm{pH} 7.4,37^{\circ} \mathrm{C}$ for 48 hours. The iron retained in the nanoparticles after 48 hours of dialysis is expressed as percentage of the amount present initially.

Abbreviations: rHDL, reconstituted high-density lipoprotein; SPION, superparamagnetic iron oxide nanoparticle; PBS, phosphate-buffered saline. treated with free valrubicin (Figure 8B). Accordingly, the $\mathrm{IC}_{50}$ values of free valrubicin and $\mathrm{rHDL}-\mathrm{SPION}$-valrubicin for PZ-HPV were found to be $22 \mu \mathrm{g} / \mathrm{mL}$ and $115 \mu \mathrm{g} / \mathrm{mL}$ (30.4 $\mu \mathrm{M}$ and $158.9 \mu \mathrm{M})$, respectively $(P$-value $<0.05)$. The potential therapeutic enhancement of the encapsulation of the therapeutic agent (valrubicin) was calculated using to the following formula:

$\begin{gathered}\begin{array}{c}\text { Valrubicin } \mathrm{IC}_{50} \\ \text { of PC-3 cells }\end{array} \\ \begin{array}{c}\text { Valrubicin } \mathrm{IC}_{50} \\ \text { of prostate epithelial cells }\end{array}\end{gathered} / \begin{gathered}\mathrm{rHDL}-\text { valrubicin } \mathrm{IC}_{50} \\ \text { of PC-3 cells }\end{gathered}$

Accordingly, the therapeutic enhancement via the rHDL-valrubicin-SPION formulation (vs free valrubicin) is indicated to be approximately sevenfold. These findings indicate substantial potential benefits during chemotherapy by limiting or avoiding off target toxicity of the rHDL/drug formulations.

The TEM data on rHDL-SPION nanoparticles before and after formulation indicated that these particles are somewhat spherical with a diameter ranging from $15 \mathrm{~nm}$ to $22 \mathrm{~nm}$ with the mean diameter of $17.9 \pm 5.1 \mathrm{~nm}$ (Figure 9A). The SPIONs before going in the formulation showed the diameter ranging from $2 \mathrm{~nm}$ to $4 \mathrm{~nm}$ with a mean diameter of $3.3 \mathrm{~nm}$ (Figure 9B).

\section{Discussion}

The present study evaluated the feasibility of preparing SPIONs in combination with rHDL nanoparticles, potentially for cancer therapeutics. The findings, reported earlier, provide additional substantial proof of concept for the utility of rHDL-SPION-valrubicin nanoparticles against prostate cancer (PC-3) cells vs nonmalignant cells and thus suggest broader application in cancer chemotherapy. Accordingly, we have shown that iron can be incorporated into the rHDL complex and that the chemical composition of rHDL-SPION 

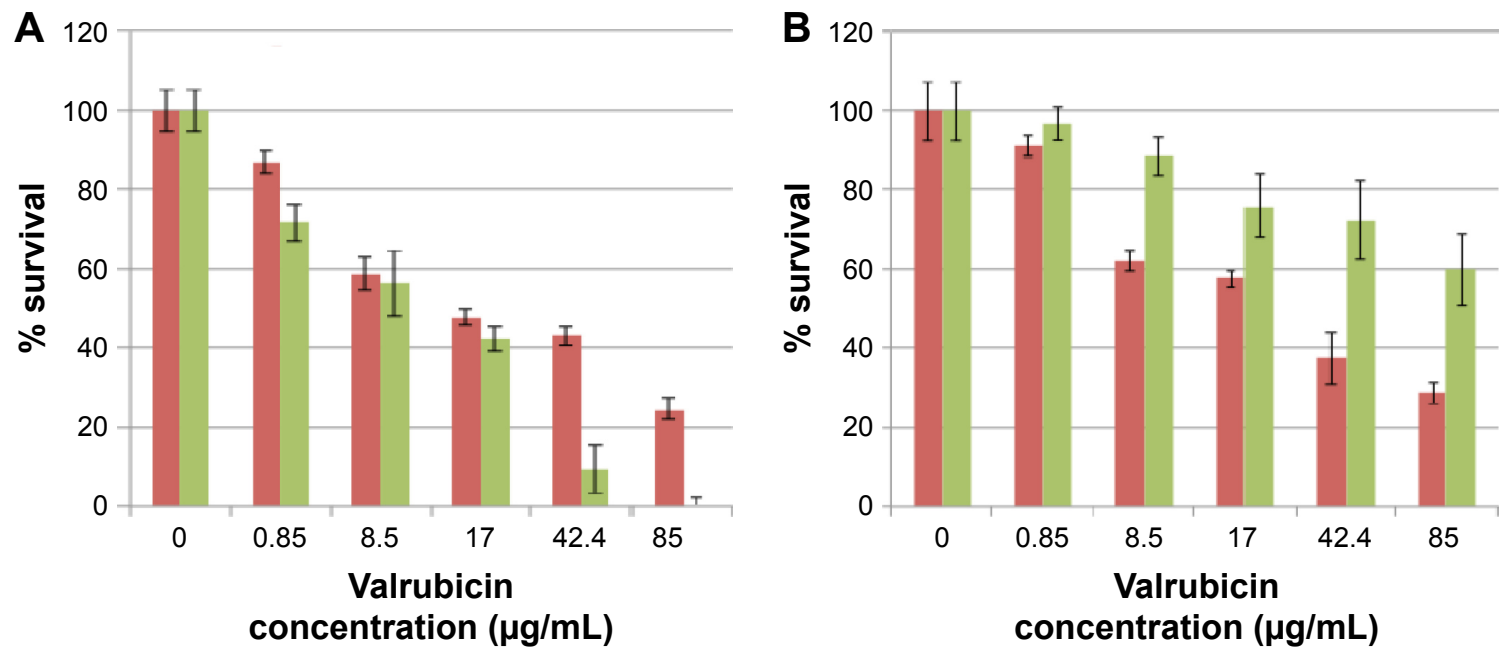

Free valrubicin rHDL-SPION-valrubicin

Figure 8 Survival of PC-3 cells (A) and PZ-HPV cells (B) upon treatment with rHDL-SPION-valrubicin vs free valrubicin and incubated for 48 hours as determined by MTT assay. Abbreviations: rHDL, reconstituted high-density lipoprotein; SPION, superparamagnetic iron oxide nanoparticle; MTT, 3-(4,5-dimethylthiazol-2-yl)-2,5-diphenyltetrazolium bromide.

nanoparticles was comparable to the rHDL-drug particles reported earlier. ${ }^{33,34}$ The protein and phospholipid contents of the rHDL-SPION valrubicin complex were comparable to those of empty rHDL and to those of the rHDL-valrubicin nanoparticles, which have been shown to be quite stable..$^{33,34}$ These findings further support the feasibility of the eventual utilization of the rHDL-SPION-valrubicin nanoparticles for clinical applications. As expected, the EE of iron and valrubicin for the rHDL-SPION-valrubicin complexes was lower than the entrapment of both rHDL-SPION and rHDLvalrubicin due to the iron particles being incorporated into the nanoparticle complex.

The size and surface properties play an important role in targeting therapeutic nanocarriers. Smaller size facilitates the nanoparticles to penetrate the tumor microenvironment and stroma more efficiently than larger structures..$^{43-45}$ The
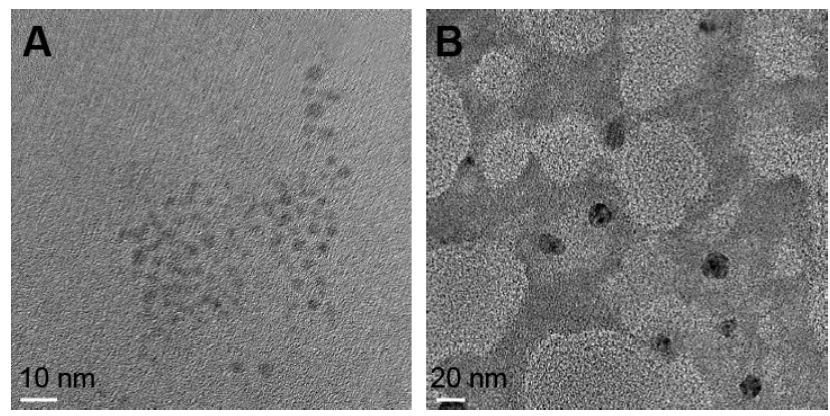

Figure 9 TEM image of rHDL-SPION nanoparticles: (A) iron oxide particles and (B) rHDL-SPION nanoparticles.

Abbreviations: TEM, transmission electron microscopy; rHDL, reconstituted high-density lipoprotein; SPION, superparamagnetic iron oxide nanoparticle. estimated diameter of rHDL-SPION particles by dynamic light scattering was $\sim 67.6 \pm 19.4 \mathrm{~nm}$, whereas with TEM, the diameter was found to be $17.9 \pm 5.1 \mathrm{~nm}$. The discrepancy between the two methods could be due to the differences in the measurements; however, the size was observed to be within the desirable range of the standard nanoparticles. ${ }^{45-48}$ Nevertheless, the size of the nanoparticles could likely be further modulated (decreased) by increasing their protein content or modifying the procedure for preparing the magnetic valrubicin rHDL formulations. The PDI for empty rHDL particles was 0.470 and for the rHDL-SPIONvalrubicin nanoparticles was 0.378 that are considered to be in excess of a desirable homogeneity. This parameter could also likely to be improved by utilizing alternate preparatory procedures for assembling the respective nanoparticles. ${ }^{49,50}$ More careful analyses of particle size and diameter will also have to be conducted to accurately assess the size and the heterogeneity of these nanoformulations. The zeta potential of rHDL-SPION-valrubicin nanoparticles $(-7.93 \mathrm{mV})$ was closer to 0 than the empty rHDL particles $(-10.57 \mathrm{mV})$, indicating that their interaction with cells may be more intense than that of empty rHDL.

BLT-1 has been shown to inhibit the function of the SR-B1 receptor, while circulating HDL competes with the rHDL nanoparticles and thus has been shown to interfere with the drug uptake from the rHDL nanoparticles. ${ }^{34,50-52}$ When the PC-3 cells were pre-incubated with BLT-1 and human HDL, respectively, and subsequently treated with rHDL-SPION and rHDL-SPION-valrubicin nanoparticles, the uptake of 
iron was reduced by almost $90 \%$, indicating that the mechanism of iron uptake was specific and likely dependent on the SR-B1 receptors. These data are also consistent with earlier findings regarding the release of the drug payload to cancer cells and tumors from the rHDL nanoparticles. ${ }^{34,42,52}$ Stability studies showed that $60 \%-65 \%$ of the iron remained within the nanoparticles after 48 hours of incubation, indicating that encasement of SPIONs and valrubicin within rHDL provided a stable environment that has the potential to remain longer in the circulation than conventional nonencapsulated drugs. Free drugs generally exit the circulation within a few hours. ${ }^{42}$ Prussian blue staining provided evidence of movement for the majority of SPIONs in the direction of the magnetic field; the accumulation of particles toward PC-3 suggest that the SPIONs inside the rHDL-drug nanoparticles may help to maneuver them toward the target site, thus facilitating the subsequent drug delivery to the cells of interest. This approach could minimize the wastage of drugs during therapy by avoiding normal healthy cells and tissues and thus reduce off-target effects.

Finally, the cytotoxicity studies support the effectiveness of rHDL-SPION-valrubicin against PC-3 over the traditional method of administering the drug in the free form. These studies are consistent with our previous observations with rHDL-valrubicin nanoparticles with PC-3 cells. ${ }^{34}$ The SPION particles are permitted to move when a magnetic field is applied, so localizing the site of attack could become even more efficient than the rHDL-encapsulated delivery. An increase in the $\mathrm{IC}_{50}$ values of $\mathrm{rHDL}-\mathrm{SPION}-$ valrubicin as compared to the free valrubicin in nonmalignant PZ-HPV cells indicates that there is a protective effect of rHDL-encapsulated drug. In vivo, this may suggest reduced side effects of the drug when delivered via the rHDL vehicle.

Although rHDL-SPION-valrubicin drug delivery model has shown a strong potential for application in cancer therapeutics, it will require significant additional work (tumor suppression, toxicology, etc) before its translation toward the clinic. One of the concerns to be addressed is the expression of SR-B1 receptor, the gateway for delivering drugs to tumors by the rHDL nanoparticles, that has been shown to be variable among cancer cells and tumors. ${ }^{53}$ This feature of tumors actually may be taken advantage of and extended toward personalized or precision therapy by pre-screening the patients' tumors for expression of the SR-B1 receptor prior to therapy to determine their susceptibility to drugs, carried by the rHDL nanoparticles. Ultimately, another concern may arise due to additional costs of the equipment and personnel to generate and monitor the magnetic field applied during therapy.

\section{Conclusion}

While the technology involving magnetic nanoparticles has been primarily investigated for the purposes of imaging, ${ }^{53-55}$ the use of rHDL-SPION-valrubicin for targeted drug delivery against malignant cells and tissues had not previously been explored. The findings of the present study show that it is feasible to formulate rHDL-SPION-drug nanoparticles for the purpose of targeted delivery of the drug payload to a desired site. Additionally, due to their properties of contrast enhancement, SPION containing rHDL particles could also be utilized as magnetic resonance imaging contrast agents, providing the rHDL-SPION-valrubicin nanoparticles with theranostic potential.

The major advantages of this technology are projected to be:

1. less toxic effects to normal cells and tissues, thus reducing the off-target effects of chemotherapy;

2. lower doses of the drug may be required to treat patients due to the more effective targeting strategy and

3. the drug payload is expected to be in circulation for a longer time, resulting in increased therapeutic effectiveness.

Subsequent to successful tumor suppression, toxicology studies and clinical trials, the inclusion of the rHDL-SPIONvalrubicin nanoparticles could provide a major advance for the chemotherapy of cancers in general and prostate cancer in particular.

\section{Acknowledgments}

This research was supported by the Cancer Prevention and Research Institute of Texas (award DP150091), Wheels for Wellness $^{\mathrm{TM}}$ (Fort Worth, TX, USA), Rutledge Foundation and Virginia Kincaid Foundation. We thank Dr Zygmunt Gryczynski for providing the spectrophotometer and fluorometer instrumentation and expert advice for the valrubicin measurements. We express our gratitude to Mr Roberto Gonzalez for his help in TEM images and Prof Jeffery Coffer, Inorganic and Materials Chemistry, Texas Christian University, Fort Worth, TX, USA, for extending the electron microscope facilities.

\section{Disclosure}

The authors report no conflicts of interest in this work.

\section{References}

1. Leong D, Rai R, Nguyen B, Lee A, Yip D. Advances in adjuvant systemic therapy for non-small-cell lung cancer. World J Clin Oncol. 2014; 5(4):633-645.

2. Liu SX, Xia ZS, Zhong YQ. Gene therapy in pancreatic cancer. World J Gastroenterol. 2014;20(37):13343-13368. 
3. McDermott D, Lebbe C, Hodi FS, et al. Durable benefit and the potential for long-term survival with immunotherapy in advanced melanoma. Cancer Treat Rev. 2014;40(9):1056-1064.

4. Heron M. Nat vital Statistics Report. Deaths: Leading Causes for 2016. 2016;65(2). Available from: http://www.cdc.gov/nchs/data/nvsr/nvsr65/ nvsr65_02.pdf. Accessed June 10, 2016.

5. National Center for Health Statistics. Health United States, 2015. Available from: https://www.cdc.gov/nchs/data/hus/hus15.pdf\#019. Accessed February 2, 2017.

6. Sriraman SK, Aryasomayajula B, Torchilin VP. Barriers to drug delivery in solid tumors. Tissue Barriers. 2014;2:e29528.

7. Au JL, Yeung BZ, Wientjes MG, Lu Z, Wientjes MG. Delivery of cancer therapeutics to extracellular and intracellular targets: determinants, barriers, challenges and opportunities. Adv Drug Deliv Rev. 2016;97:280-301.

8. Cun X, Chen J, Ruan S, et al. A novel strategy through combining iRGD peptide with tumor-microenvironment-responsive and multistage nanoparticles for deep tumor penetration. ACS Appl Mater Interfaces. 2015; 7(49):27458-27466.

9. Nel AE, Madler L, Velegol D, et al. Understanding biophysicochemical interactions at the nano-bio interface. Nat Mater. 2009;8(7): 543-557.

10. Facciabene A, Peng X, Hagemann IS, et al. Tumour hypoxia promotes tolerance and angiogenesis via CCL28 and T(reg) cells. Nature. 2011 475(7355):226-230.

11. Durymanov MO, Rosenkranz AA, Sobolev AS. Current approaches for improving intratumoral accumulation and distribution of nanomedicines. Theranostics. 2015;5(9):1007-1020.

12. Simonsen TG, Gaustad JV, Leinaas MN, Rofstad EK. High interstitial fluid pressure is associated with tumor-line specific vascular abnormalities in human melanoma xenografts. PLoS One. 2012;7(6):e40006.

13. Ishida $\mathrm{T}$, Kiwada $\mathrm{H}$. Alteration of tumor microenvironment for improved delivery and intratumor distribution of nanocarriers. Biol Pharm Bull. 2013;36(5):692-697.

14. Song C, Zhu S, Wu C, Kang J. Histone deacetylase (HDAC) 10 suppresses cervical cancer metastasis through inhibition of matrix metalloproteinase (MMP) 2 and 9 expression. J Biol Chem. 2013;288(39): 28021-28033.

15. Chen KL, Bothun GD. Nanoparticles meet cell membranes: probing nonspecific interactions using model membranes. Environ Sci Technol. 2014;48(2):873-880.

16. Ferrari M. Cancer nanotechnology: opportunities and challenges Nat Rev Cancer. 2005;5(3):161-171.

17. Tao JJ, Visvanathan K, Wolff AC. Long term side effects of adjuvant chemotherapy in patients with early breast cancer. Breast. 2015;24(suppl 2) S149-S153.

18. Xu L, Zhou DS, Zhao J, et al. Long-term therapy with sorafenib is associated with pancreatic atrophy. J Surg Res. 2015;199(2):314-321.

19. SG Komen [webpage on the Internet]. Short-Term Side Effects of Chemotherapy. Available from: http://ww5.komen.org/BreastCancer/ShortTermSideEffectsofChemotherapy.html. Accessed February 8, 2016.

20. Chemocare [webpage on the Internet]. What Are the Short and Long Term Side Effects of Chemotherapy? Available from: http://chemocare. com/chemotherapy/what-is-chemotherapy/what-are-the-long-andshort-term-effects.aspx. Accessed February 8, 2016.

21. Dehkordi A, Heydarnejad MS, Fatehi D. Quality of life in cancer patients undergoing chemotherapy. Oman Med J. 2009;24(3):204-207.

22. van der Kloot WA, Uchida Y, Inoue K, et al. The effects of illness beliefs and chemotherapy impact on quality of life in Japanese and Dutch patients with breast or lung cancer. Chin Clin Oncol. 2016;5(1):3.

23. Bae KH, Chung HJ, Park TG. Nanomaterials for cancer therapy and imaging. Mol Cells. 2011;31(4):295-302.

24. Bazak R, Houri M, El Achy S, Kamel S, Refaat T. Cancer active targeting by nanoparticles: a comprehensive review of literature. $J$ Cancer Res Clin Oncol. 2015;141(5):769-784.

25. Minko T, Rodriguez-Rodriguez L, Pozharov V. Nanotechnology approaches for personalized treatment of multidrug resistant cancers. Adv Drug Deliv Rev. 2013;65(13-14):1880-1895.
26. Alexander-Bryant AA, Vanden Berg-Foels WS, Wen X. Bioengineering strategies for designing targeted cancer therapies. Adv Cancer Res. 2013; 118:1-59.

27. Neuberger T, Schöpf B, Hofmann H, Hofmann M, von Rechenberg B. Superparamagnetic nanoparticles for biomedical applications: possibilities and limitations of a new drug delivery system. J Magn Mater. 2005; 293:483-496.

28. Hajba L, Guttman A. The use of magnetic nanoparticles in cancer theranostics: toward handheld diagnostic devices. Biotechnol Adv. 2016; 34(4):354-361.

29. Lacko AG, Nair M, Paranjape S, Mooberry L, McConathy WJ. Trojan horse meets magic bullet to spawn a novel, highly effective drug delivery model. Chemotherapy. 2006;52(4):171-173.

30. Sabnis N, Lacko AG. Drug delivery via lipoprotein-based carriers: answering the challenges in systemic therapeutics. Ther Deliv. 2012;3(5): 599-608.

31. Zhang Z, Chen J, Ding L, et al. HDL-mimicking peptide-lipid nanoparticles with improved tumor targeting. Small. 2010;6(3):430-437.

32. Lou B, Liao XL, Wu MP, Cheng PF, Yin CY, Fei Z. High-density lipoprotein as a potential carrier for delivery of a lipophilic antitumoral drug into hepatoma cells. World J Gastroenterol. 2005;11(7): 954-959.

33. McConathy WJ, Nair MP, Paranjape S, Mooberry LK, Lacko AG. Evaluation of synthetic/reconstituted high-density lipoproteins as delivery vehicles for paclitaxel. Anticancer Drugs. 2008;19(2): 183-188.

34. Sabnis N, Nair M, Israel M, McConathy WJ, Lacko AG. Enhanced solubility and functionality of valrubicin (AD-32) against cancer cells upon encapsulation into biocompatible nanoparticles. Int $J$ Nanomedicine. 2012;7:975-983.

35. Shah SA, Chib R, Raut S, et al. Photophysical characterization of anticancer drug Valrubicin in rHDL nanoparticles and its use as an imaging agent. Biophys J. 2015;108(2):623a.

36. Raut S, Kirthivasan B, Bommana M, Squillante E, Sadoqi M. The formulation, characterization and in vivo evaluation of a magnetic carrier for brain delivery of NIR dye. Nanotechnology. 2010;21(39): 395102-395112.

37. Department of Chemistry, University of Kentucky. Experiment 5: Molecular Absorption Spectroscopy: Determination of Iron with 1,10-Phenanthroline. CHE 226 - Analytical Chemistry Laboratory, Department of Chemistry, University of Kentucky; 2005. Available from: http://www.chem.uky.edu/courses/che226/labs/050-fe_absorption.pdf. Accessed September 27, 2014.

38. Life Science Products and Services. Prussian Blue Staining Protocol for Iron. Available from: http://www.ihcworld.com/_protocols/ special_stains/prussian_blue.htm. Accessed December 15, 2014.

39. Yang CR, Zhao XL, Hu HY, et al. Preparation, optimization and characteristic of huperzine a loaded nanostructured lipid carriers. Chem Pharm Bull (Tokyo). 2010;58(5):656-661.

40. Arnould R, Dubois J, Abikhalil F, et al. Comparison of two cytotoxicity assays - tetrazolium derivative reduction (MTT) and tritiated thymidine uptake - on three malignant mouse cell lines using chemotherapeutic agents and investigational drugs. Anticancer Res. 1990;10:145-154.

41. Leary J. The importance of zeta potential for drug/gene delivery in nanomedicine. In: Malvern Instruments Workshop. West Lafayette, IN: Purdue University; 21 September 2011. Guest Lecture.

42. Mooberry LK, Nair M, Paranjape S, McConathy WJ, Lacko AG. Receptor mediated uptake of paclitaxel from a synthetic high density lipoprotein nanocarrier. J Drug Target. 2010;18(1):53-58.

43. Naahidi S, Jafari M, Edalat F, Raymond K, Khademhosseini A, Chen P. Biocompatibility of engineered nanoparticles for drug delivery. J Control Release. 2013;166(2):182-194.

44. Bricarello DA, Smilowitz JT, Zivkovic AM, German JB, Parikh AN. Reconstituted lipoprotein: a versatile class of biologically-inspired nanostructures. ACS Nano. 2011;5(1):42-57.

45. Malvern [webpage on the Internet]. Zeta potential. Malvern Instruments Ltd.; n. d. Available from: http:/www.malvern.com/en/products/ measurement-type/zeta-potential/. Accessed September 27, 2014. 
46. Murugan K, Choonara YE, Kumar P, Bijukumar D, du Toit LC, Pillay V. Parameters and characteristics governing cellular internalization and trans-barrier trafficking of nanostructures. Int J Nanomedicine. 2015;10: 2191-2206.

47. Faraji AH, Wipf P. Nanoparticles in cellular drug delivery. Bioorg Med Chem. 2009; 17(8):2950-2962.

48. Wiesenthal A, Hunter L, Wang S, Wickliffe J, Wilkerson M. Nanoparticles: small and mighty. Int J Dermatol. 2011;50(3):247-254.

49. Sheridan C. Proof of concept for next-generation nanoparticle drugs in humans. Nat Biotechnol. 2012;30(6):471-473.

50. Pattni BS, Chupin VV, Torchilin VP. New developments in liposomal drug delivery. Chem Rev. 2015;115(19):10938-10966.

51. Nieland T, Penman M, Dori L, Krieger M, Kirchhausen T. Discovery of chemical inhibitors of the selective transfer of lipids mediated by the HDL receptor SR-BI. Proc Natl Acad Sci U S A. 2002;99(24): $15422-15427$.
52. Sabnis N, Pratap S, Akopova I, Bowman PW, Lacko AG. Pre-clinical evaluation of rHDL encapsulated retinoids for the treatment of neuroblastoma. Front Pediatr. 2013;1:6.

53. Shahzad MM, Mangala LS, Han HD, et al. Targeted delivery of small interfering RNA using reconstituted high-density lipoprotein nanoparticles. Neoplasia. 2011;13(4):309-319.

54. Mao X, Xu J, Cui H. Functional nanoparticles for early detection of cancer. Wiley Interdiscip Rev Nanomed Nanobiotechnol. 2016;8(6): 814-841.

55. Panagiotopoulos N, Duschka RL, Ahlborg M, et al. Magnetic particle imaging: current developments and future directions. Int J Nanomedicine. 2015;22(10):3097-3114.

\section{Publish your work in this journal}

The International Journal of Nanomedicine is an international, peerreviewed journal focusing on the application of nanotechnology in diagnostics, therapeutics, and drug delivery systems throughout the biomedical field. This journal is indexed on PubMed Central, MedLine, CAS, SciSearch ${ }^{\circledR}$, Current Contents ${ }^{\circledR} /$ Clinical Medicine,
Journal Citation Reports/Science Edition, EMBase, Scopus and the Elsevier Bibliographic databases. The manuscript management system is completely online and includes a very quick and fair peer-review system, which is all easy to use. Visit http://www.dovepress.com/ testimonials.php to read real quotes from published authors. 